\title{
Activity and Health Status Monitoring System
}

\author{
Stefan Oniga \\ IT Systems and Networks \\ University of Debrecen \\ Debrecen, Hungary \\ oniga.istvan@inf.unideb.hu
}

\author{
Alin Tisan \\ Computing and Technology \\ Anglia Ruskin University \\ Cambridge, UK \\ alin.tisan@anglia.ac.uk
}

\author{
Robert Bólyi \\ Intelligent Embedded Systems Laboratory \\ University of Debrecen \\ Debrecen, Hungary Róbert Bólyi \\ bolyirobi@gmail.com
}

\begin{abstract}
Physical activity monitoring represents an important tool in supporting/encouraging vulnerable persons in their struggle to recover from surgery or long term illness promoting a healthy lifestyle. The paper proposes a smart, low power activity monitoring platform capable to acquire data from 4 inertial sensor modules placed on human body, temporarily store it on a mobile phone for real time data display or on a server for long term data analysis.
\end{abstract}

Keywords-Physical activity, Monitoring platforms; Inertial sensors; Ambient Intelligence, Ubiquitous Health, Smart Cities

\section{INTRODUCTION}

Physical inactivity is a significant, independent risk factor for a range of long-term health conditions affecting society today. Monitoring the physical activity/inactivity of vulnerable people: disabled, elderly, persons with long term illnesses under continuous medical treatment, postoperative patients represents a priority for maintaining a high quality of life or for supporting patients recovering from surgery and/or identifying possible health complications providing substantial reduction in healthcare costs.

Being a key health indicator, many studies has been dedicated into finding reliable monitoring systems which can give information about activity/inactivity of a subject over a long period of time. The studies reported in the literature investigate: (i) the sensors for monitoring specific activities with single or multiple inertial sensors as in [1-3,18], or biosensors [4], (ii) monitored activity: sit, stand, lay, walk, fall, stairs ascend/descend, (iii) sensors body positioning [5,15,17] or activity-specific estimation methods [6].

Besides these, another important topic is the affordability of the monitoring systems and it seems that finding reliable lowcost devices is still a challenging issue at the moment.

Due to its cost and performance, accelerometers are the most used sensors in physical activity (PA) monitoring and recognition. They provide body movement information over 3 axes, from which it can be inferred the tilt, speed and distances. However, as the recording storage is limited, features of the activity are usually extracted. The most common features are extracted in the time domain such as mean, variance, mean absolute variance, min-max, zero crossing rate [7-10], etc. or in frequency domain where spectral energy, entropy or principal frequency are calculated using Fast Fourier Transform and Wavelet Transform $[8,9,11]$.

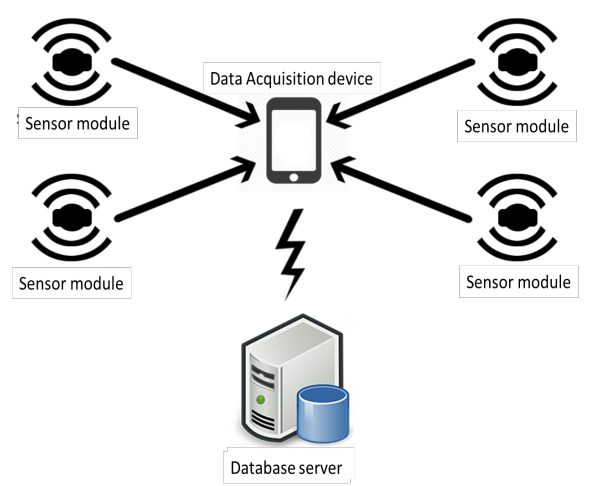

Fig. 1. Activity and Health Status Monitoring System Architecture

In this paper it is described an activity monitoring system that acquires and stores data generated by 4 wearable devices (tags) which consists of accelerometers and gyroscopes. The sensorial modules are worn on the wrists and ankles for best activity data acquisition. Data is initially acquired on a mobile phone through Bluetooth Low Energy (BLE) and later sent to a database server over WIFI for visualization and analysis.

The rest of the paper is organized as in the following: in section II the architecture of the monitoring system is presented followed in section III by the measurement results and conclusions.

\section{SYSTEM'S ARCHITECTURE}

The system proposed consists of 3 main components: (i) Sensor modules, (ii) Data acquisition module and (iii) Database server, as depicted in Fig. 1. It follows the 3 layer IoT structure with a sensing layer, a network layer and an analysing layer.

\section{A. The sensor module}

The sensor module used for the monitoring system is Bytereal RealTag 1.0 shown in Fig.2. The module is fit in a 3D printed wearable plastic case. This permits the sensor module to be attached to the wrist or ankle using an elastic strap.

The sensor module is a power optimized CC2541 BLE low power System on Chip (SoC) module developed by Texas Instrument which consists of an Intel 8051 microprocessor, a 2.4-GHz Bluetooth Low Energy module and a InvenSense MPU6050 MEMS sensor with 6 degrees of freedom which combines a 3 axes accelerometer, 3 axes gyroscope and a Digital Motion Processor (DMP). 


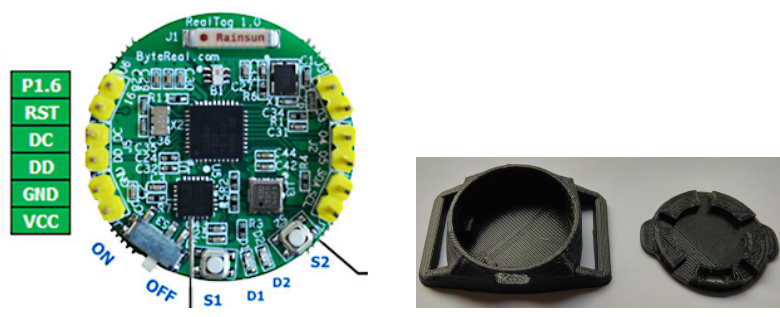

Fig. 2. Sensor module

Also, the module is equipped with a 12-Bit ADC with eight channels and configurable resolution, battery monitor module and sleep timers making it suitable for applications which require a very low power consumption [13].

\section{B. Data acquisition app}

For setting the sensor activity (sleep or active mode), data acquisition from the sensors and data storage on the database server, a mobile app on an Android platform has been developed. The mobile phone is used as an intermediate layer for buffering the data generated by the sensors before loading it on the database server.

The app has implemented following components: 3 Activities, 2 Services, 1 Content Provider and 1 Broadcast Receiver as depicted in Fig. 3:

The initial setting of the system requires module sensors pairing with the mobile app done through the interface created (Fig 4) and their connectivity to the phone/app, as shown in Fig. 5 where the MAC addresses of the modules can be observed.

\section{Database server}

The database web server stores the data acquired from the sensors for further data access and monitoring activities, user data ID and sensor modules' ID and name. The graphical user interface created on the webserver, permits user login, data access and export in .xlsx or .csv data type for data processing with specialized software such as Matlab or Labview.

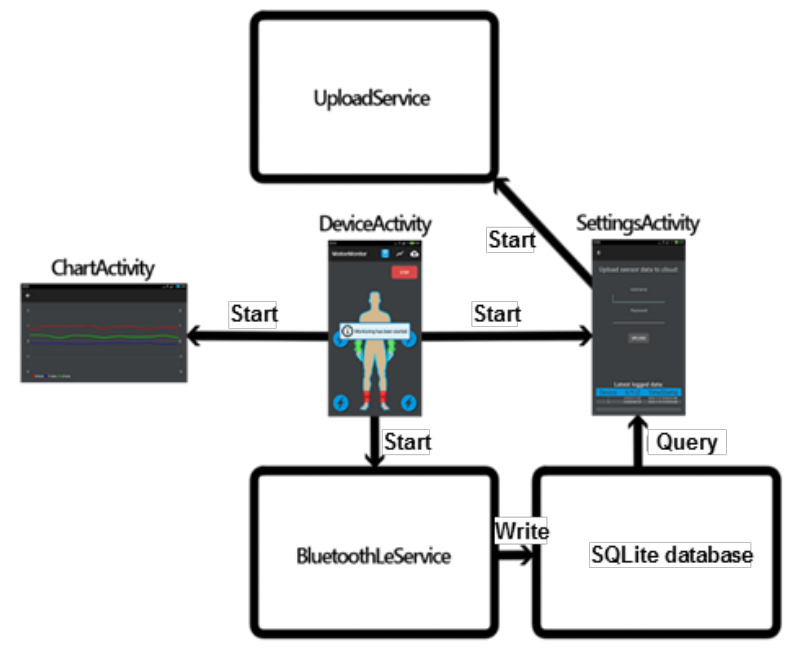

Fig. 3. The block diagram of the data acquisition system
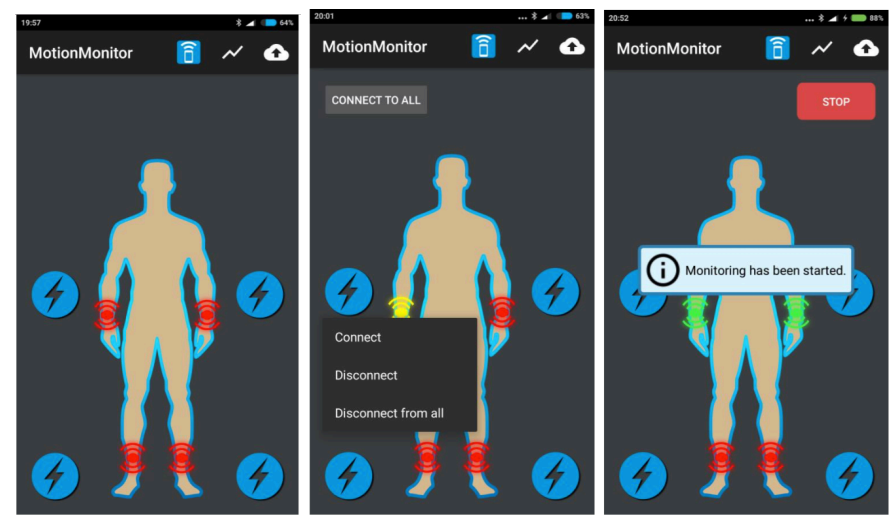

Fig. 4. Mobile phone app interface for sensor modules connection

The implemented functions permit data processing for feature extraction and display.

The algorithms used for the implemented features extraction were calculated in time domain: mean, variance, RMS, Kurtosis (1) and min-max or frequency domain: Spectral energy (2), Spectral Entropy (3), Principal frequency (4), Spectral centroid. In the following equations $T$ indicates the window size, $F$ is the number of frequency components, $i$ refers to the accelerometer dimensions $(x, y, z), a_{i}(t)$ is an element of time series and $A i(f)$ is a frequency component. A good comparison of the features extraction algorithms can be found in [15].

Mean, variance, mean absolute deviation and root mean square are statistical indicators which give information about sample distribution.

Kurtosis (1) measures the peak-ness of probability distribution of collected data.

$$
K S_{i}=\frac{\frac{1}{T} \sum_{t=1}^{T}\left(a_{i}(t)-M_{i}\right)^{4}}{\left(\frac{1}{T} \sum_{t=1}^{T}\left(a_{i}(t)-M_{i}\right)^{2}\right)^{2}}
$$

Min-max is the difference between the maximum and the minimum values of time series.

Generally, signal energy is the area under the squared signal. In this case, SE measures the sum of the squared frequency components. (Since, the spectrum is symmetric, in the following three formulas $F$ can be replaced by $F / 2$ ).

$$
S E_{i}=\sum_{f=1}^{F}\left|A_{i}(f)\right|^{2}
$$

The entropy is a measure for uncertainty. The following formula is the entropy of the normalized power spectrum density.

$$
E_{i}=-\sum_{f=1}^{F} \frac{\left|A_{i}(f)\right|^{2}}{\sum_{j=1}^{F}\left|A_{i}(j)\right|^{2}} \log _{2}\left(\frac{\left|A_{i}(f)\right|^{2}}{\sum_{j=1}^{F}\left|A_{i}(j)\right|^{2}}\right)
$$




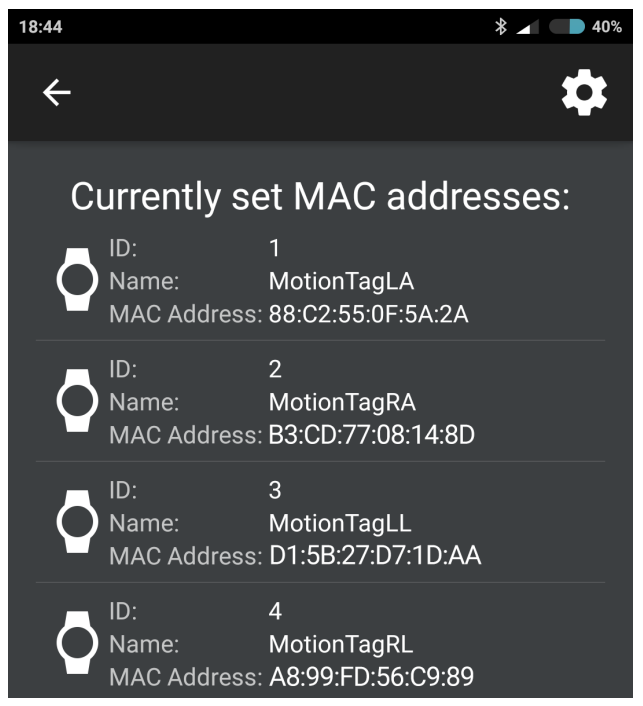

Fig. 5. Sensors MAC address setting

Principal frequency refers to the most significant frequency component which has the highest amplitude (DC component was omitted).

$$
P F_{i}=\max _{f}\left|A_{i}(f)\right| \quad f \neq 0
$$

The database is built on the open source PostgreSQL objectrelational database system. The database diagram of the monitoring system is shown in Fig. 6. It consists of 3 tables: USER_ACCOUNT table which records user's ID, MEASUREMENTS table which stores the acquired data and DEVICE table that records the sensor modules' ID and name.

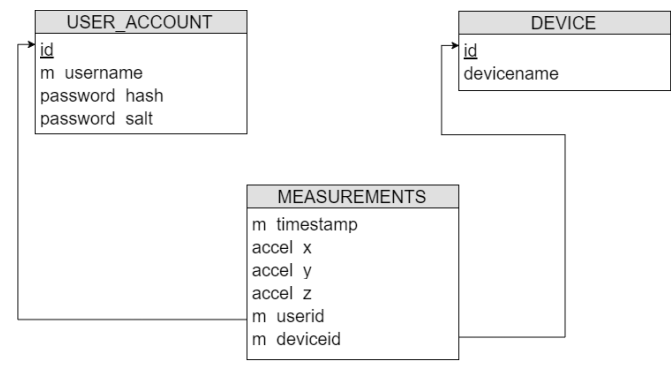

Fig. 6. The database diagram of the monitoring system

The chosen runtime environment for the webserver is Node.js. The system is open source, cross-platform JavaScript Runtime Environment that allows the developing of a wide range of Javascript applications and tools. The Node.js brings the world of event-driven programming to the web server development, allowing rapid development of applications [14].

The created graphical user interface has 3 main components: (i) User login/registration, (ii) Data selection and (iii) Data visualization. In Fig. 7 it is presented the control tabs/fields for setting the user login, the representation type, the sensor module and the recorded data.

\section{DATA MEASUREMENT}

The structure of the data acquired consists in a matrix of 4 rows (where 4 is the number of sensors used) and 7 columns

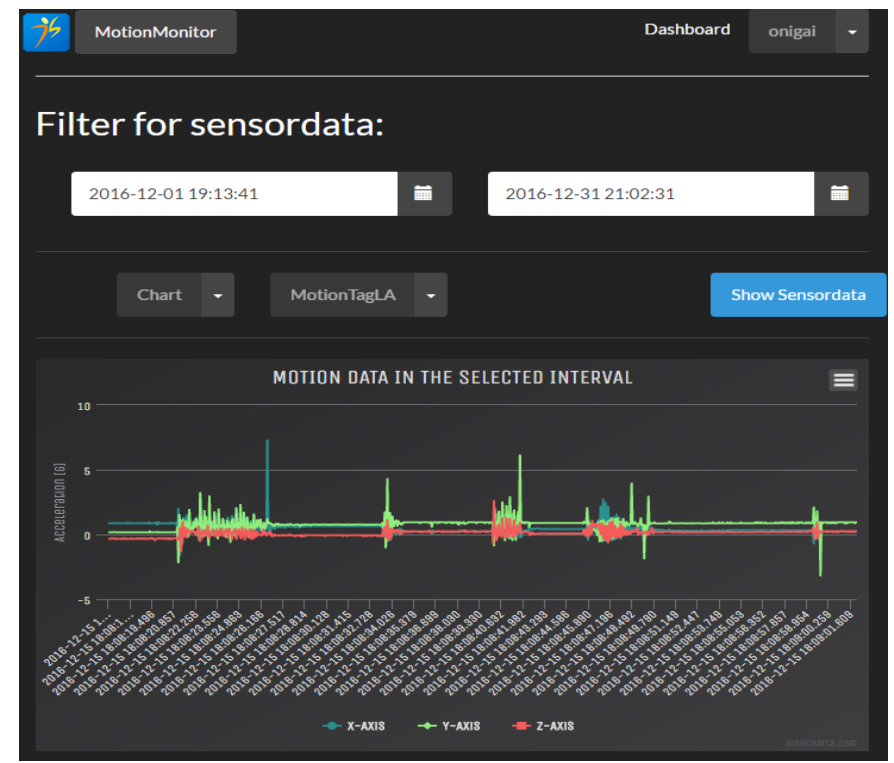

Fig. 7. The acquired data visualization

representing the time stamp $(\mathrm{t})$, accelerometer data on 3 axes, $\left(a_{x}\right),\left(a_{y}\right),\left(a_{z}\right)$ and gyroscope data on 3 axes $\left(g_{x}\right),\left(g_{y}\right)$ and $\left(g_{z}\right)$. The device ID values were set to 1 for the module worn on the left arm, 2 for the left leg, 3 for the right arm and 4 for the right leg. For the presented application only the accelerometers data have been used, remaining that the gyroscopes data to be acquired/analysed in a following application.

The sensor modules are supplied from batteries, therefore, power consumption represents an important parameter for a remote measurement system. Considering the frequencies likely to be recorded for the activities monitored (walk, sleep, fall), the acquisition sampling rate was set to $20 \mathrm{~Hz}$. Moreover, for extending the life battery, the sensor modules were set in the sleep mode in between the measurements. Considering the settings above, the power consumed by the sensor modules has been measured considering 3 operating modes: sleep mode (the sensor module is in between the measurements and it is waiting to be waken-up), data measuring mode (the 3 axes acceleration and gyroscope values are calculated and sent over BLE to the mobile app) and broadcasting (mobile phone- sensor modules pairing process). The power consumption values measured are presented in table I. The sensor modules are supplied from a button cell dioxide CR2032 battery with a capacity of $225 \mathrm{mAh}$ which permits the sensor modules to function continuously for period of 16 hours ( 2 days with 8 h per day).

Table I. Current consumption per operating mode

\begin{tabular}{|c|c|c|}
\hline Operating mode & Time & Current consumption \\
\hline broadcast & $5 \mathrm{~ms}$ every $100 \mathrm{~ms}$ & $20 \mathrm{~mA}$ \\
\hline measurement & $10 \mathrm{~ms}$ every $50 \mathrm{~ms}$ & $5 \mathrm{~mA}$ \\
\hline sleep & $40 \mathrm{~ms}$ & $1 \mathrm{us}$ \\
\hline
\end{tabular}

A set of data comprising different PA: jog (samples 0 to 400), frequent sittings (samples 400 to 600 and 1400 to 2000), walk (samples 600 to 1400) has been recorded and presented in Fig. 10. On the acquired data feature extraction algorithms (Matlab coded) in time domain (Fig. 11 and 12) and frequency domain (fig 13) have been applied. It can be observed that the 


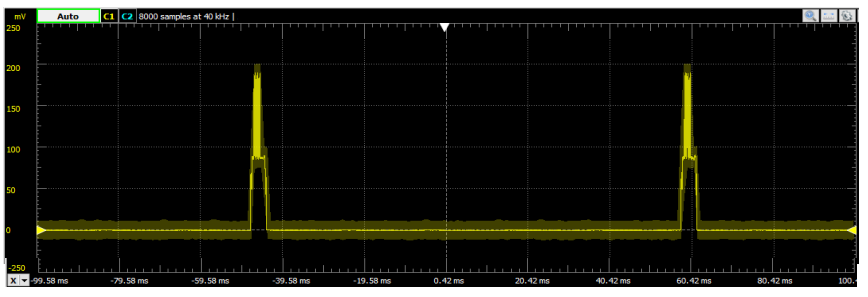

Fig. 8. Sensor module power consumption during pairing

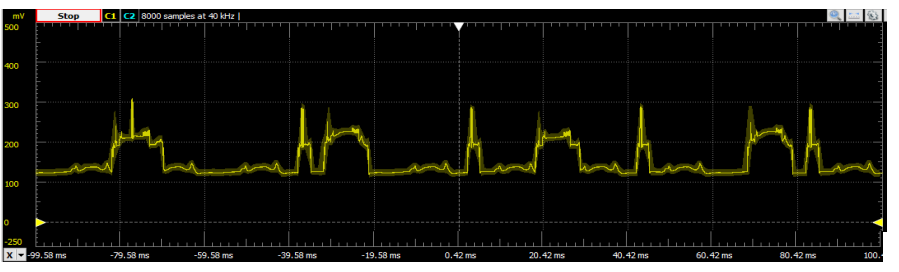

Fig. 9. Sensor module power consumption during measurement mode (the horizontal axis represents the voltage drop on a $10 \mathrm{ohms}$ resistor)

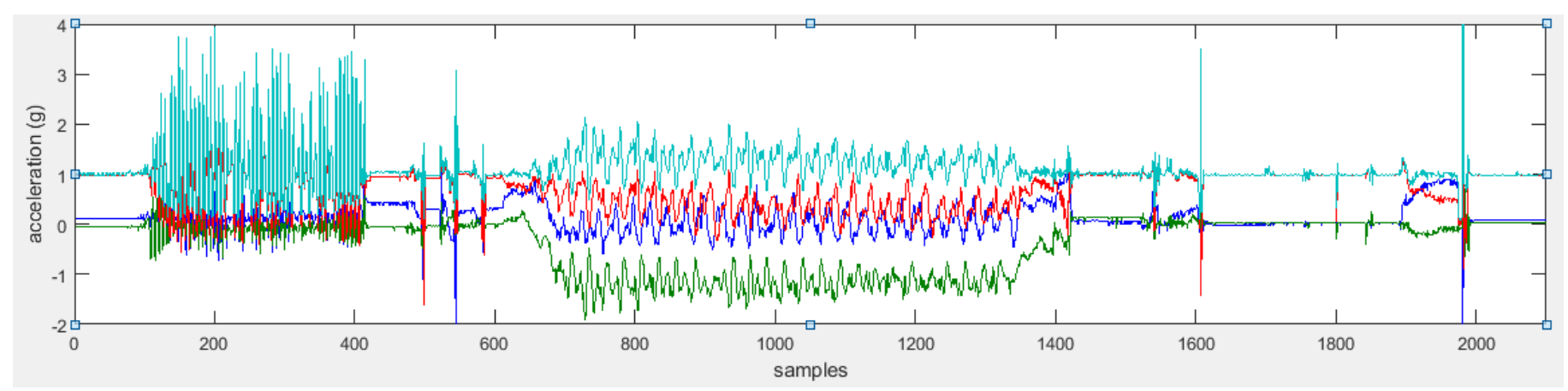

Fig. 10. Activity recording and display
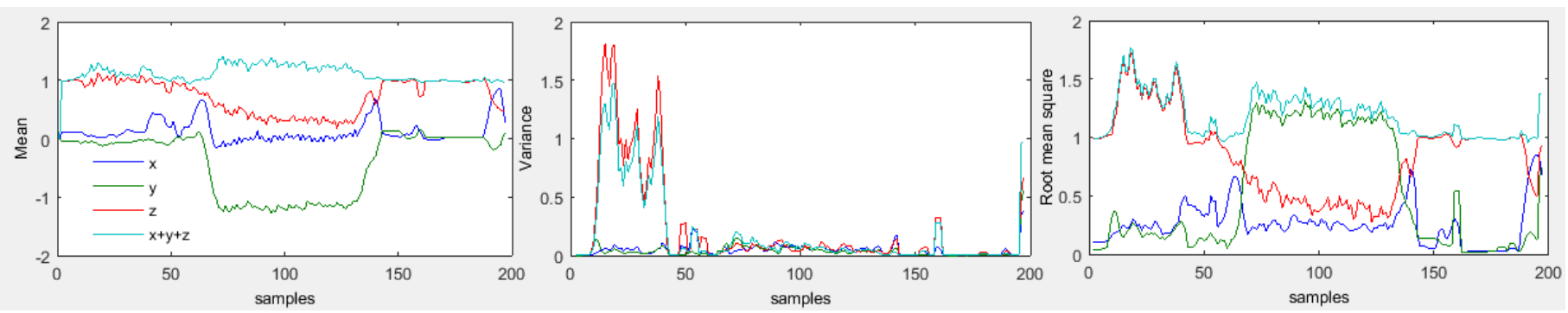

Fig. 11. Time domain features extraction (mean, variance, RMS)
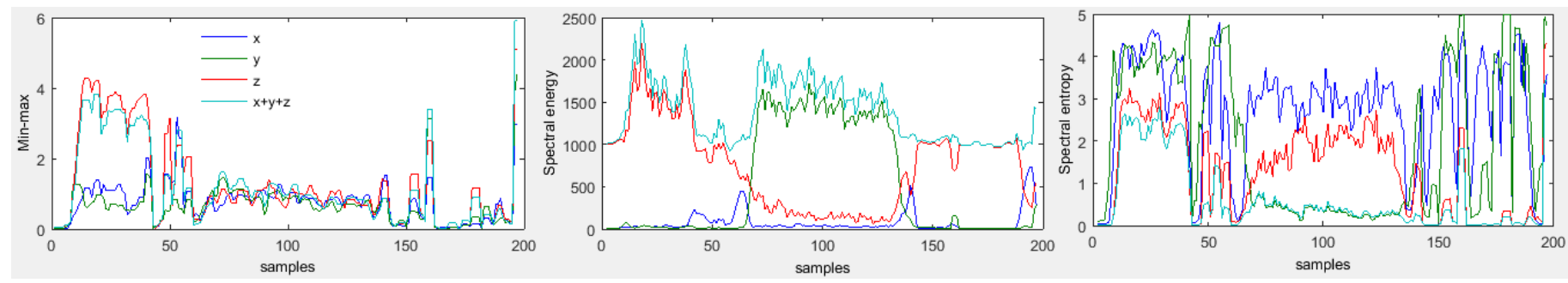

Fig. 12. Time and frequency domain features extraction (Min-max, Spectral energy, Spectral Entropy)
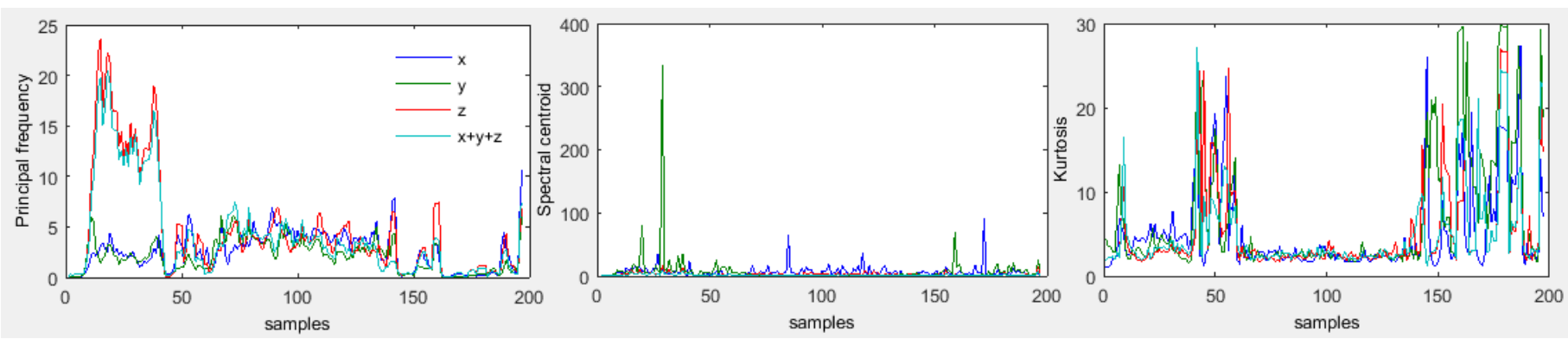

Fig. 13. Time and frequency domain features extraction (Principal frequency, Spectral centroid, Kurtosis) 
features extracted from the raw signal preserve the activity information and allow easy recognition of the PA evolved (by feeding the features extracted as inputs to an artificial neural network).

\section{CONCLUSIONS}

In this paper we present an activity and health status monitoring system using 4 motion sensor tags, a mobile phone and an app as an acquisition hub and database server for data storage, visualization and export. The database created permits the monitoring of physical activities of persons under medical surveillance offering information about the recovery/recovery status. The physical activities can be latter recognized by implementing pattern recognition algorithms using artificial neural networks as the one presented in [11]. In comparison with the system presented in [12], the present monitoring system has the advantage of a lower size, much more comfortable to be worn and does not need special acquisition system but a normal intelligent mobile phone.

\section{REFERENCES}

[1] Khan, Adil Mehmood, et al. "A triaxial accelerometer-based physicalactivity recognition via augmented-signal features and a hierarchical recognizer."Information Technology in Biomedicine, IEEE Transactions on 14.5 (2010): 1166-1172.

[2] Biswas, Dwaipayan, et al. "Recognizing upper limb movements with wrist worn inertial sensors using k-means clustering classification." Human movement science 40 (2015): 59-76.

[3] Kwapisz, Jennifer R., Gary M. Weiss, and Samuel A. Moore. "Activity recognition using cell phone accelerometers." ACM SigKDD Explorations Newsletter, (2011): 74-82.

[4] Naik, Ganesh R., and Dinesh Kant Kumar. "Twin SVM for gesture classification using the surface electromyogram." Information Technology in Biomedicine, IEEE Transactions on 14.2 (2010): 301308.

[5] Atallah, Louis, et al. "Sensor positioning for activity recognition using wearable accelerometers." Biomedical Circuits and Systems, IEEE Transactions on 5.4 (2011): 320-329.
[6] M. Altini, J. Penders and O. Amft,"Energy expenditure estimation using wearable sensors: A new methodology for activity-specific models," in Proc. Wireless Health Conf., 2012

[7] Qin Ni, Ana Belén García Hernando, et al, The Elderly’s Independent Living in Smart Homes: A Characterization of Activities and Sensing Infrastructure, Sensors 2015, 15, pp.11312-11362, ISSN 1424-8220

[8] Y. Zheng, W. K. Wong, X. Guan, S. Trost, Physical activity recognition from accelerometer data using a multi-scale ensemble method. In Proceedings of the 25th Conference on Innovative Applications of Artificial Intelligence, Bellevue, Washington, USA, 14-18 July 2013.

[9] H. Gjoreski, M. Gams, Accelerometer data preparation for activity recognition. In Proceedings of the International Multiconf. Information Society, Ljubljana, Slovenia, 10-14 October 2011; pp. 10-14.

[10] M. Jiang, H. Shang, Z. Wang, H. Li, Y. Wang, A method to deal with installation errors of wearable accelerometers for human activity recognition, Physiol. Meas. 2011, 32, 347-358.

[11] I. Orha,S. Oniga, Study regarding the optimal sensors placement on the body for human activity recognition, 2014 IEEE 20th International Symposium for Design and Technology in Electronic Packaging (SIITME), 23-26 Oct. 2014, pp. 203-206.

[12] I. Orha, S. Oniga, "Real time data acquisition system for activity recognition using motion sensors," Carpathian Journal of Electronic and Computer Engineering Volume 9/2, 2016, pp. 18-21.

[13] Texas Instruments Technical Documents - CC2514 2.4-GHz Bluetooth $^{\mathrm{TM}}$ low energy and Proprietary System-on-Chip

[14] Pedro Teixeira, Professional Node.js: Building Javascript Based Scalable Software, John Wiley \& Sons, 2012

[15] S. J. Preece, J. Y. Goulermas, L. P. J. Kenney, D. Howard, “A comparison of different feature generation methods in activity classification," in Proc. Int. Conf. Ambul. Monit. Phys. Act. Movement, Rotterdam, The Netherlands, 2008, p. 87.

[16] Chun Zhu, W. Sheng, Motion-and location-based online human daily activity recognition, Pervasive Mob. Comput. 2011, 7, 256-269.

[17] A. Mannini, S. S. Intille, M. Rosenberger, A. M. Sabatini, W. Haskell, Activity recognition using a single accelerometer placed at the wrist or ankle. Med. Sci. Sports Exerc. 2013, 45, 2193-2203.

[18] L. Gao, A. K. Bourke, J. Nelson, Evaluation of accelerometer based multi-sensor versus single-sensor activity recognition systems. Med. Eng. Phys. 2014, 36, 779-78 